\title{
The Energy-Consumption of CPU and Memory Prediction in Cloud Computing Based on GREY-ARIMA Model
}

\author{
Yong Shao, Yuxiang Zhang ${ }^{+}$, Changshun Yan and Shengchang Wang \\ ${ }^{1}$ College of Software Engineering, Beijing University of Technology, Beijing 100124, China
}

\begin{abstract}
The energy-consumption of data center in cloud computing is a heated issues. This paper focuses on the challenges of operation and power management in cloud platform, and presents a GMARIMA model, which is simultaneously exploiting autoregressive integrated moving average(ARIMA model) and GM(1,1) model. This combination is used for CPU and Memory in the cloud computing experiment to model, test and forecast. The experiments revealed the GM-ARIMA model, in the minimum amount of history record, is an efficient and feasible model for the limited sampled forecasting.
\end{abstract}

Keywords: cloud computing, Grey-ARIMA model, the consumption of CPU and memory, prediction research.

\section{Introduction}

Nowadays, with the rapidly developing of the Internet, in order to handle mass data and improve the efficiency of service, the technology of cloud computing comes into being. However, due to the randomness task of cloud computing platform, sparse and dense degree of different machine work is different, which causes the existence of spare resources wasting [1]. The prediction of resource consumption's status becomes a vital factors to improve the efficiency of cloud computing platform [2], [3]. Therefore, the prediction of data center in cloud computing has a significant value to research.

\section{Related Work}

Some scholars research and propose a kind of forecasting method of cloud computing load resources matched with the mode in this field, such as Caron, et al. ${ }^{[2]}$, and this method used improving the string matching algorithms (KMP). Saripalli, et al. ${ }^{[5]}$ proposed a method to identify the resources load peak and forecasting.. Prevost, et al. ${ }^{[6]}$ use the neural network and self-regression model to forecast the loads of different types of resources.

However, the main principle of these methods is forecast data by tracking and matching a large amount of historical data, and for the new built data center in cloud computing, which has insufficient historical data, is not suitable for these methods.

To improve this deficiency, based on the traditional ARIMA model, this article uses the residual error amendment method of GM $(1,1)$ model to establish a combination model to get the forecasting result and improve the accuracy of ARIMA model.

\section{Establishment of Gray Combination Model}

\subsection{GM(1,1) model}

\footnotetext{
+ Corresponding author Yuxiang Zhang. Tel.: +86 18612866698

E-mail address: $583110401 @$ qq.com
} 
The gray system is belonged to the small sample analysis, compared with the growth rate analysis method, only above 4 data can establish the model [7] and it is more convenient. $\mathrm{GM}(1,1)$ model is the basic model [8] of gray forecasting theory, and realizes the correct derivation of system operation law after researching part of information to get the future forecasting value [9].

The $\operatorname{GM}(1,1)$ model prediction formula is, setting the original sequence

$$
\begin{gathered}
\mathrm{X}^{(0)}=\left(x^{(0)}(1), x^{(0)}(2), \ldots x^{(0)}(n)\right), \text { then } \\
\mathrm{x}^{(0)}(\mathrm{k})=a^{(1)} x^{(1)}(k)=x^{(1)}(k)-x^{(1)}(k-1), \quad k=1,2 \ldots, n
\end{gathered}
$$

\subsection{ARIMA model}

Autoregressive Integrated Moving Average Model (ARIMA) is a famous time sequence forecasting method proposed by Box and Jenkins in the early 1970s [7]. The so-called ARIMA model is a model that the non-stable time sequence converts to the stable time sequence, and then regress the lagged value of dependent variability and present value and lagged value of random error item.

The ARIMA model prediction formula is

$$
L_{t}=\varphi_{1} Y_{t-1}+\varphi_{2} Y_{t-2}+\cdots+\varphi_{p} Y_{t-p}+e_{t}-\theta_{1} e_{t-1}-\theta_{2} e_{t-2}-\cdots-\theta_{q} e_{t-q}
$$

\subsection{GM-ARIMA model}

In order to improve the forecasting accuracy of model, this article is to improve ARIMA model by residual error amendment method, forecast the residual error sequence value of ARIMA model via GM(1,1) model, and add the forecasting result in the original forecasting value of ARIMA model to improve the residual error and increase the forecasting.

Set a time sequence $\{x(i), i=1,2, \ldots, n\}$ used ARIMA model get predictive value $\hat{x}^{(0)}(i)$. Set a time sequence $\left\{\mathrm{e}^{(0)}(L)\right\}$ is the residual error of ARIMA model, then use $\operatorname{GM}(1,1)$ model to $\left\{\mathrm{e}^{(0)}(L)\right\}$ get the predictive sequence $\widehat{X}^{(0)}(i, 1)$, the combined model's formula is,

$$
\widehat{X}^{(0)}(i, 1)=\widehat{X}^{(0)}(i)+\hat{e}^{(0)}(1)
$$

\section{Experimental Analysis}

The data of this case is from the real data of Cloud Computing Center Laboratory of a company in Beijing, due to this laboratory is newly built and doesn't have enough historical data, so using Grey-ARIMA model to forecast the utilization of CPU and memory.

\subsection{Data Processing}

Screen the peak time data of CPU and memory in experiment center firstly, for the machine with high use rate of CPU and machine with low use rate of memory. The CPU data has the characteristics of mutability, due to the laboratory didn't run data once. And the memory data has characteristics of stability. By those data, we can verify the applicability of Grey-ARIMA model in mutable sequence and stable sequence.

Using ARIMA model to forecast CPU use rate and memory use rate to get the forecasting sequence of CPU and memory use rate. And then calculate to get the corresponding the residual error sequence. Use the method in Section 3.1 to establish the gray GM $(1,1)$ model for the residual error sequence of number, and Figure $1 \& 2$ show the trend of ARIMA model and $\operatorname{GM}(1,1)$ combination model.

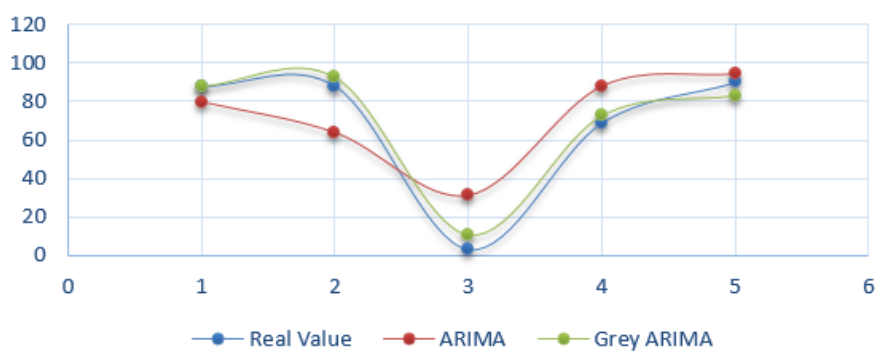

Fig. 1: The prediction of CPU. 


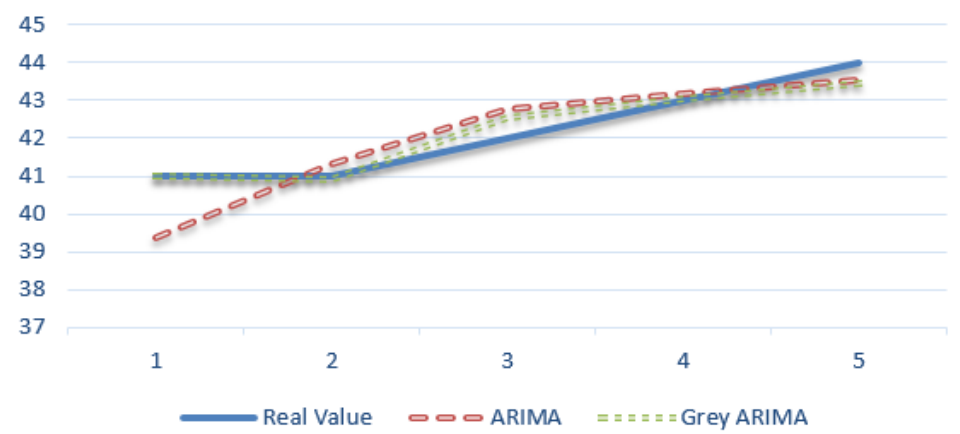

Fig. 2: The prediction of memory.

Substitute the data into the formula MSE $=\sqrt{\frac{1}{n} \sum_{i=1}^{n}\left|\left(Y_{i}-\widehat{Y}_{i}\right)\right|}$ for calculating the mean square error; And get error of average absolute percentage by formula MAE $=\frac{1}{n} \sum_{i=1}^{n}\left|\frac{Y_{i}-\vec{Y}_{i}}{Y_{i}}\right|$ The comparison results are in Table 1.

Table 1: Comparison of precision of ARIMA model and gray ARIMA model

\begin{tabular}{lllll}
\hline & CPU_MSE & CPU_MAE & MEMORY_MSE & MEMORY_MAE \\
\hline ARIMA & 1.952 & $204 \%$ & 0.395 & $16.1 \%$ \\
Grey-ARIMA & 1.359 & $75.5 \%$ & 0.382 & $1.2 \%$ \\
Reduce error & 0.594 & $55.1 \%$ & 0.013 & $14.9 \%$ \\
\hline
\end{tabular}

\subsection{Analysis of Grey-ARIMA model}

The experiment result reveal that Grey-ARIMA model predict the output the curve is closer to the actual value, on the handling of the small amount of data, and, the predicted performance of Grey-ARIMA model is better than the classic ARIMA model.

According to the data in Table 1, in the forecasting value of CPU use rate, you can see that the mean square error of surface gray ARIMA model reduces 0.594, and the average absolute percentage error reduces $55.1 \%$. In the forecasting of memory use rate, the mean square error of surface gray ARIMA model reduces 0.013 , and the average absolute percentage error reduces $14.9 \%$.

Furthermore, according to the characteristics of the data sequence about the utilization of CPU and memory, and Grey-ARIMA model for the two sequence can guarantee high accuracy of predicted results, show that this model has strong applicability in mutable sequence and stable sequence.

\section{Conclusion}

Compared to the classic forecasting model which need a lot of historical data, Grey-ARIMA model can provide powerful support to the newly established enterprises. The experimental results also proved that Grey-ARIMA model, in the field of the hardware energy-consumption predicted in cloud computing, can effectively improve the predicted accuracy, correct the defect of single forecasting method, has a strong applicability and great prospect. In the future, we can consider in combination Grey-ARIMA model with load balanced algorithm related cloud computing, study in the field of load balanced in cloud computing.

\section{Reference}

[1] Tan YM,Zeng GS, Wang W. Policy of Energy Optimal Management for Cloud Computing Platform with Stochastic Tasks[J]. Ruan Jian Xue Bao/ Journal of Software,2012,23(2):266-278.(in chinese)

[2] Changbo Ke, Ruchuan Wang, Fu Xiao, and Zhiqiu Huang, "Requirement-Oriented Privacy Protection Analysis Architecture in Cloud Computing," Journal of Communications, vol. 10, no. 1, pp. 55-63, 2015. Doi: $10.12720 / \mathrm{jcm} .10 .1 .55-63$

[3] Ye KJ, Wu ZH, Jiang XH, He QM. Power Management of Virtualized Cloud Computing Platform[J]. Chinese Journal of Computers,2012,35(5):1262-1285(in Chinese) 
[4] Caron,E.,Desprez,F.,Muresan,A.Forecasting for Gid and Cloud Computing On-Demand Resources Beased on Pattern Matching[C].2nd IEEE Int.Conf.on Cloud Computing Technology and Science,2010:456-463

[5] Saripalli,P.,et al.Load Prediction and Hot Spot Detection Models for Autonomic Cloud Computing[C].4th IEEE International Conference on Utility and Cloud Computing.2011:397-402

[6] Prevost J J,et al.Predictionof cloud data center networks loads using stochastic and neural models[C].6th International Conference on Systems Engineering (SoSE),2011:276-281

[7] Deng J L.Control problems of grey systems[J].Systems \& Control Letters, 1982,1(5):288-294

[8] Deng J L.Grey control systems[M].Wuhan:Press of Huazhong University of Science and Technology,1985

[9] Liu S F, Lin Y.Grey Systems:theory and application[M]. London: Springer 2011

[10] G. E. P. Box and G. M. Jenkins. Time Series Analysis: Forecasting and Control. San Francisco: Holden Day, 1976 